\title{
Measurement of the Ambient and Extremity Doses in Clinical Oncology Hospital, Menoufia University, Egypt
}

\author{
I. A. El-Mesady1", G.M. Saleh², N. E. Khaled³, A. A. Hussein'1, H. M. El-Samman1, Kh. K. Abd-Al Aziz ${ }^{2}$ \\ ${ }^{1}$ Physics Department, Faculty of Science, Menoufia University, Egypt \\ ${ }^{2}$ Clinical Oncology and Nuclear Medicine Department, Faculty of Medicine, Menoufia University, Egypt \\ ${ }^{3}$ Ionizing Radiation Department, National Institute for Standards, Giza, Egypt \\ Email: *intesar_sah2001@yahoo.co.uk
}

How to cite this paper: El-Mesady, I.A., Saleh, G.M., Khaled, N.E., Hussein, A.A., El-Samman, H.M., Abd-Al Aziz, Kh.K. (2018) Measurement of the Ambient and Extremity Doses in Clinical Oncology Hospital, Menoufia University, Egypt. International Journal of Medical Physics, Clinical Engineering and Radiation Oncology, 7, 248-260.

https://doi.org/10.4236/ijmpcero.2018.72021

Received: April 14, 2018

Accepted: May 27, 2018

Published: May 30, 2018

Copyright $\odot 2018$ by authors and Scientific Research Publishing Inc. This work is licensed under the Creative Commons Attribution International License (CC BY 4.0).

http://creativecommons.org/licenses/by/4.0/

\begin{abstract}
The ambient dose of radiation therapy and nuclear medicine units of Clinical Oncology Hospital, Menoufia University were investigated using thermoluminescence dosimeter MTS-700 and surveymeter (Inspector Radiation Alert). The maximum\% difference between read out of both MTS-700 (TLD) and surveymeter did not exceed $6 \%$ and $8 \%$ for the two hospital units respectively. Values of the annual ambient dose received in both hospital units were found to be incompliant with radiation protection regulations. In addition, the extremity effective dose Hp (0.07) of staff in nuclear medicine unit was measured using wrist and finger techniques. Results indicate in-homogenies distribution of fingertips doses. Radiation doses received by the wrists and fingertips of radiopharmaceutical staff preparing ${ }^{99 \mathrm{~m}} \mathrm{Tc}$ syringe were observed to be higher by a factor of about 1.41 and 1.44 respectively than those for the administrating staff whom injecting patients by ${ }^{99 \mathrm{~m}} \mathrm{Tc}$ syringe, but also still in congruent with international radiation protection regulations.
\end{abstract}

\section{Keywords}

Ambient Dose, Extremity Dose, Thermoluminescence, Lithium Fluoride Dosimeter

\section{Introduction}

The use of ionizing radiations widely applied, especially in the context of medical diagnostics and therapy as well as for material testing and many other purposes [1] [2]. Radiation has been used in medicine. Between 30 and 50 percent of medical decisions, especially the critical ones, are made after studying the results 
of radiological examinations [3] [4]. In medicine, the most important requirement is the radiation dosimetry for surveillance of radiation workers in radiology, nuclear medicine and radiation oncology. In addition, its importance was defined for quality assurance (i.e. Precise estimation of delivering dose to the patient) in both external beam radiation therapy and brachytherapy as well [5]. Because of the large and growing number of patients undergoing radiation therapy and the multiple nuclear medicine procedures, continued efforts are required to improve the quality of treatment, diagnosis and to reduce the associated radiological risk [6]. So, multiple therapeutic and diagnostic procedures in radiation therapy and nuclear medicine units of Clinical Oncology Hospital, Menoufia University, Egypt require proper attention.

In the present work, an ambient radiation monitoring program is assessed. Knowledge of ambient dose values is relevant to the occupational exposure personnel who may limit the time spent at high dose areas. Recommendations were given to the departmental authorities to implement actions in order to reduce doses at high dose sites in order to comply with the ALARA principle [7]. This will be discussed whether the exposed doses at the work places are acceptably and safe or not. The investigated sites at radiation therapy unit are a radiation therapy clinic, ${ }^{60}$ Cocontrol, patient waiting, long hall, worker path room, linear accelerator control, simulator control and ${ }^{60} \mathrm{Co}$ treatment room. Moreover, other selected sites in the nuclear medicine unit are chosen, physician station, technician station, X-ray room, injection room, preparing material, patient path room, external hall and patient waiting room. In addition, determination and investigation of the effective skin dose at depth $0.07 \mathrm{~mm}(\mathrm{Hp}(0.07))$ of the body of medical and paramedical staff in the nuclear medicine unit will be done using wrist and finger MTS-700 (TLD). Special attention has been paid to the exposure of nuclear medicine worker hands. The radio pharmacists who label various ligands can be exposed to high radiation doses to their fingertips (primarily of the thumb, index finger and middle finger). Quite frequently, the $\mathrm{Hp}(0.07)$ to the fingertips of those three fingers may exceed the dose limit, i.e. value of 500 $\mathrm{mSv} / \mathrm{y}$ for the skin of human fingers; this dose limit refers to the maximum dose recorded [8].

\section{Methodology}

\subsection{Experimental Procedure for Ambient Dose Measurements}

In radiation therapy unit some radiation sources are in use; these are ${ }^{60} \mathrm{Co}$ (Cobalt-60) gamma source (Theratronics 780-E (1.25 MeV). This teletherapy unit was manufactured in Canada by Theratronics International Limited for Oncology System and used for radiation therapy treatment of cancer patients. $\mathrm{X}$-ray Photon beam from medical linear accelerator (Varian Clinac 600-C with nominal energy $6 \mathrm{MeV}$ ) was used in radiation therapy unit for radiation therapy treatment of cancer patients. This device provides rectangular, symmetric and asymmetric fields and generates therapeutically useful megavoltage X-rays with 
exceptional flexibility, control, precision, and reproducibility). In addition, X-ray beam from radiotherapy simulator(Ximatron C-series) with an energy range of $(40-150 \mathrm{kVp})$ and serial number 99,599 manufactured in the U.S.A. by VARIAN Associated Limited for Oncology System was used in radiation therapy unit. It was used for simulating the treatment radiation fields before and during radiation therapy treatment of cancer patients). In nuclear medicine unit, ${ }^{99 \mathrm{~m}} \mathrm{Tc}$ (Technetium-99) generator with activity of $50 \mathrm{GBq}(1351.35 \mathrm{mCi})$ and energy of gamma $140 \mathrm{keV}$ and X-ray machine (General Electric Healthcare)with energy range $(40-120 \mathrm{kVp}$ )are also in use. For many years the most commonly used TLDs were LiF detectors doped with $\mathrm{Mg}$ and $\mathrm{Ti}$ [9]. Sintered pellets of the same chemical composition produced in Poland from natural LiF are referred under the trade name MTS-N [10] [11]. MTS-700 (TLD) referred to that produced from enriched 7Li isotopes will be used in the measurements. MTS-700 indicates the accumulated dose, which divided by the number of measurement hours to give dose rate. MTS-700 is tissue equivalent, its $Z_{\text {eff }}$ (effective atomic number) is 8.13 [12]. In order to investigate the dosimetric characteristics of MTS-700 (TLD), ${ }^{60} \mathrm{Co} \gamma$-source with dose rate $=48.08 \mathrm{cGy} / \mathrm{min}$ and radiation field size of $10 \times 10 \mathrm{~cm}^{2}$ was used. MTS-700 (TLD) readings will be compared with results read out directly by surveymeter (Inspector Radiation Alert) manufactured by SE International, INC serial number $(21,083)$ underwent mandatory annual calibration by National Institute of Standard, NIS Egypt. The inspector is a Geiger-Müller detector, the most useful and famous radiation detector consists of a microprocessor controlled radiation measuring instrument which offers excellent sensitivity to low levels of alpha, beta, gamma and X-rays. The digital readout is displayed with a red count light and audible beep. Providing instant indications of the radiation level. Other benefits include an adjustable timer and external calibration control [13]. The exposure rate in $\mathrm{mR} / \mathrm{h}$ was converted into absorbed dose rate in $\mathrm{mG} / \mathrm{h}$ using a standard conversion. Figure 1 and Figure 2 show,

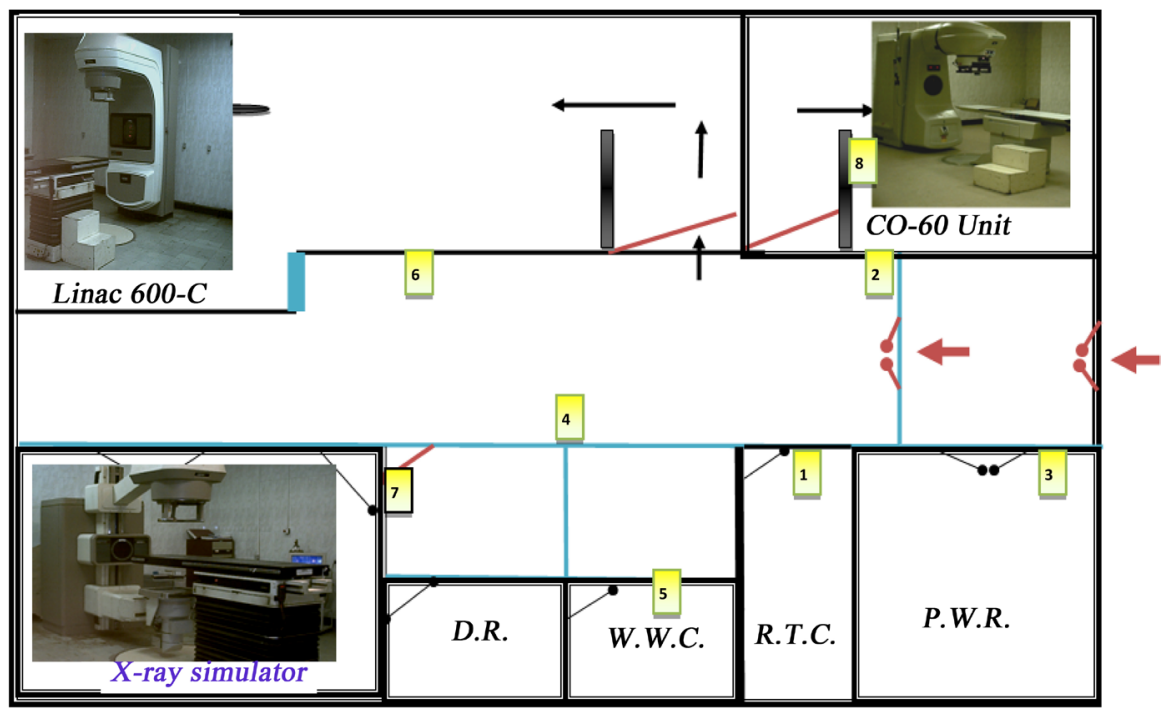

Figure 1. Layout of MTS-700 dosimeters in radiation therapy unit. 


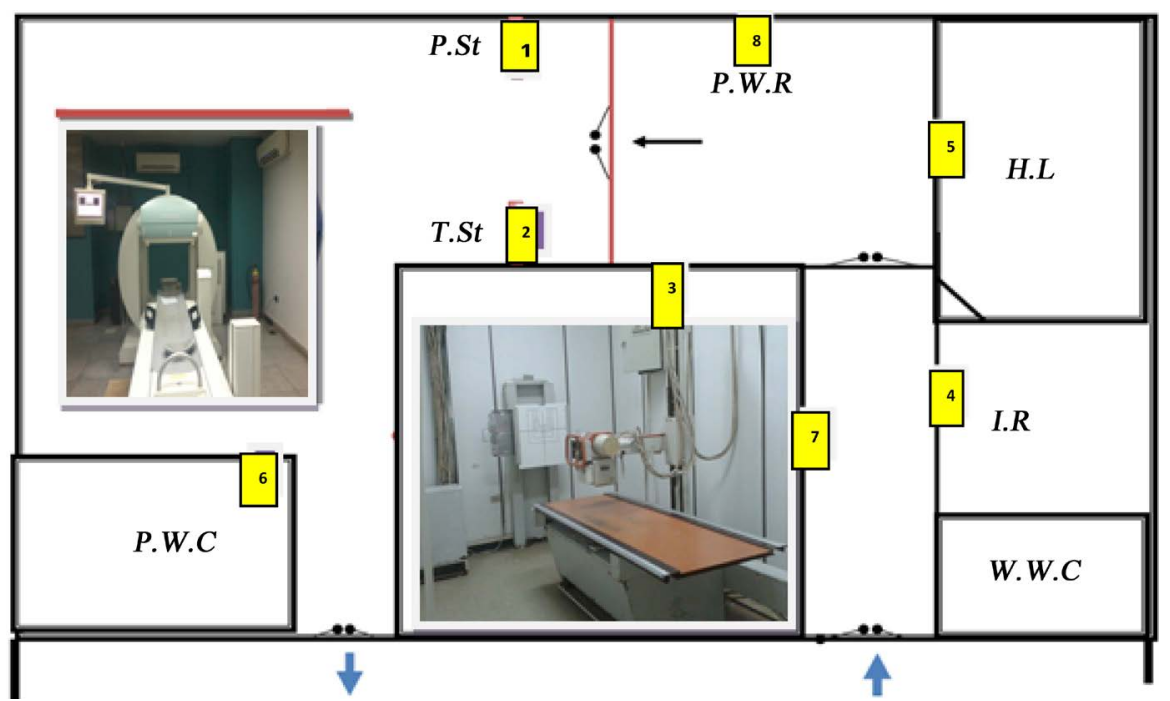

Figure 2. Layout of MTS-700 dosimeters in nuclear medicine unit.

respectively the layout of MTS-700 dosimeters in radiation therapy and nuclear medicine units. The surveymeter and TL dosimeters were fixed on $120 \mathrm{~cm}$ height from the ground, at 8 different locations, two chips of MTS-700 (TLD) in each defined position. TLD read out was taken after exposure by one day to fade the low temperature peaks using Harshaw bicorn model 3500 TL reader

\subsection{Experimental Procedure for Extremity Dose Measurements of Medical and Paramedical Staff in Nuclear Medicine Unit}

Nuclear medicine implies the manipulation of unsealed radioactive sources. Labelle ${ }^{99 \mathrm{~m}} \mathrm{Tc}$ in hospital divided into three steps kit preparation, dispensing syringes and administration as described in Table 1. The two steps No. 1 and 2 were performed by physicists or technologists and step No. 3 performed by physicians or nurses, according to a department rotation periodic work table for nuclear medicine unit workers. In this research, the workers did so by volunteering for their colleague, the second author, to complete his Ph.D. They were divided into two groups, the first group called preparing radiopharmaceuticals group (physicists) and the second group called injecting radiopharmaceuticals group (nurses). In nuclear medicine procedures, radiation exposure of hands, especially in fingertips, is much higher than that of the thorax. [14] [15]. If the dose to any part of the extremities of a worker is likely to exceed three tenths of the annual dose limit, an additional dosimeter should be placed on the part of the extremity where the dose is expected to have its highest value. In practice, extremity monitoring is carried out by measuring the personal dose equivalent Hp (0.07) [16]. The work load includes numbers of patients and the scan type during one week the period of investigation described in Table 2. Two pairs of gloves equipped with eight chips of MTS-700 (TLD) were fixed in a certain position as in Figure 3 in a palm side of the two hands of the two groups at the centers of the wrists and fingertips of middle, index, and thumb fingers. 
Table 1. Steps of ${ }^{99 \mathrm{~m}}$ TcLabelle in the nuclear medicine unit at Menoufia University, Egypt.

\begin{tabular}{|c|c|c|}
\hline \multirow{2}{*}{$\begin{array}{l}\text { Steps of }{ }^{99 \mathrm{~m}} \mathrm{Tc} \\
\text { Labelle No. }\end{array}$} & \multicolumn{2}{|c|}{$\begin{array}{l}\text { Process and Procedures in the nuclear medicine unit } \\
\text { at Menoufia University, Egypt }\end{array}$} \\
\hline & Process & Procedures \\
\hline \multirow{3}{*}{1} & \multirow{3}{*}{ Kit Preparation } & 1) Eluting ${ }^{99 \mathrm{~m}} \mathrm{Mo}-{ }^{99 \mathrm{~m}} \mathrm{Tc}$ generator in elute vial (50 GBq). \\
\hline & & 2) Determining specific activity in dose calibrator. \\
\hline & & 3) Drawing from elution vial and adding saline \\
\hline \multirow{3}{*}{2} & \multirow{3}{*}{ Dispensing Syringes } & 1) Dispensing in syringes from kit vial. \\
\hline & & 2) Checking activity in dose calibrator. \\
\hline & & 3) Transfer of unshielded syringes to lead transport box. \\
\hline \multirow[b]{2}{*}{3} & \multirow[b]{2}{*}{ Administration } & 1) Remove needle and mounting syringe shield. \\
\hline & & $\begin{array}{l}\text { 2) Inject patient with } 10 \mathrm{~mm} \text { saline to check the cannula } \\
\text { before inject with radiopharmaceutical and again injected } \\
\text { with } 10 \mathrm{~mm} \text { saline after radiopharmaceutical for washing. }\end{array}$ \\
\hline
\end{tabular}

Table 2. Number of patients and the scan type during the investigation, one week in the nuclear medicine unit at Menoufia University, Egypt.

\begin{tabular}{ccc}
\hline \multirow{2}{*}{ No. } & \multicolumn{2}{c}{ Number of patients and the scan type during the investigation } \\
\cline { 2 - 3 } & Number of patients & Scan type \\
\hline 1 & 55 & Bone scan \\
3 & 18 & Cardiac scan \\
4 & 4 & Thyroid scan \\
& 11 & Renal scan \\
\hline
\end{tabular}

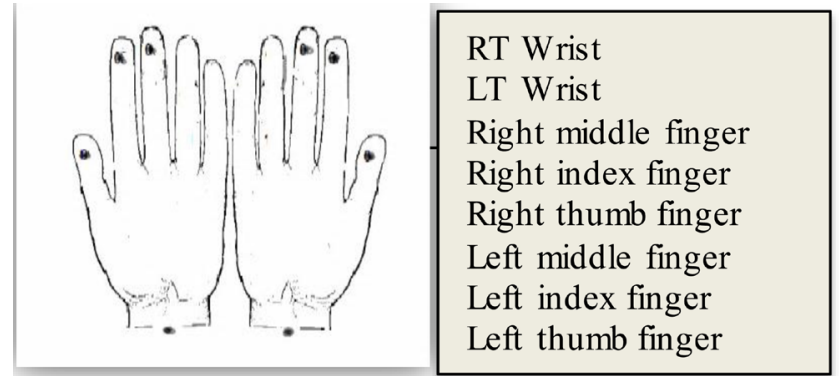

Figure 3. Distribution and Positions of MTS-700 (TLDs) in the palm side of the right and the left hands during preparing and injecting ${ }^{99 \mathrm{~m}}$ Tc persons.

\section{Results}

The element correction coefficient (ECC) was calculated for a set of measurements carried out on 15 pieces from MTS-700 (TLD) irradiated at dose of 2 Gy The mean value of relative ECF was found to be ranged between 0.96 and 1.04 with standard deviation (SD) 0.02 and coefficient of variance (CV) $2.28 \%$ (see Table 3). In order to assess the repeatability of the dose measurements attainable using the MTS-700 (TLD) a set of repeated readouts, 15 chips for each cycle, 
Table 3. The range, mean, SD, and CV of relative ECC for MTS-700 (TLD).

\begin{tabular}{|c|c|}
\hline MTS-700 (TLD) No. & Element Correction Coefficient \\
\hline 1 & 1.018 \\
\hline 2 & 0.988 \\
\hline 3 & 1.003 \\
\hline 4 & 0.981 \\
\hline 5 & 0.977 \\
\hline 6 & 1.004 \\
\hline 7 & 1.022 \\
\hline 8 & 1.030 \\
\hline 9 & 1.008 \\
\hline 10 & 0.970 \\
\hline 11 & 1.015 \\
\hline 12 & 1.036 \\
\hline 13 & 0.955 \\
\hline 14 & 1.004 \\
\hline 15 & 1.007 \\
\hline Range & $0.995-1.036$ \\
\hline Mean & 1.001 \\
\hline SD & 0.02 \\
\hline $\mathrm{CV}$ & $2.28 \%$ \\
\hline
\end{tabular}

was carried out at dose $2 \mathrm{~Gy}$. The mean value of relative sensitivity in seven cycles of irradiations was found to be ranged between 0.98 and 1.01 with (SD) range $0.022-0.047$. This indicates good stability of the crystals in the TLD batch. Therefore, reproducibility with (SD) 0.03 and (CV) $3.21 \%$ over 105 TL signal readings became satisfactory regarding to the International Electrotechnical Commission, IEC requirements for environmental and personal dosimetry [17] [18]. The dosimeters showed high resistance to adverse effects caused by handling procedures (see Table 4). Variance in sensitivity of a typical batch of TL dosimeters is unavoidable but can be reduced from $10 \%-15 \%$ to $1 \%-2 \%$ when dosimeters are calibrated [19]. A calibration factor ( $\mathrm{nC} / \mathrm{mGy}$ ) was assigned to each one. This process of calibration was repeated for two times to seven groups of MTS-700 (TLD). Each group contains two dosimeters irradiated with different radiation doses from $100 \mathrm{mGy}$ up to $2000 \mathrm{mGy}$ at the constant time, temperature profile (TTP) equal $10^{\circ} \mathrm{C} / \mathrm{s}$ to check the linearity and reproducibility of MTS-700 (TLD) response. Linear response was observed within the irradiated doses the coefficient of determination $\left(\mathrm{R}^{2}\right)$ obtained from a linear regression analysis was 0.9983 (see Figure 4 and Figure 5).

The results of ambient dose measurements using MTS-700(TLD) and inspector radiation alert (surveymeter) for radiation therapy and nuclear medicine 
Table 4. The mean, \pm SD and Coeff. of Var. of relative TL signals for MTS-700 (TLD) as irradiated seven cycles at 2 Gy Co-60 $\gamma$-rays.

\begin{tabular}{|c|c|c|c|}
\hline \multirow{2}{*}{ Cycle No. } & \multicolumn{3}{|c|}{$\begin{array}{l}\text { The Mean, } \pm \text { Standard Deviation and Coefficient of Variation of relative TL } \\
\text { signals for MTS-700(TLD) as irradiated seven cycles at } 2 \mathrm{~Gy}{ }^{60} \mathrm{Co} \gamma \text {-rays. }\end{array}$} \\
\hline & Mean & $\pm \mathrm{SD}$ & $\mathrm{CV}$ \\
\hline 1 & 0.980 & 0.027 & 2.79 \\
\hline 2 & 1.000 & 0.039 & 3.89 \\
\hline 3 & 1.010 & 0.025 & 2.44 \\
\hline 4 & 1.010 & 0.047 & 4.65 \\
\hline 5 & 1.000 & 0.023 & 2.29 \\
\hline 6 & 1.010 & 0.022 & 2.21 \\
\hline 7 & 1.000 & 0.042 & 4.21 \\
\hline
\end{tabular}

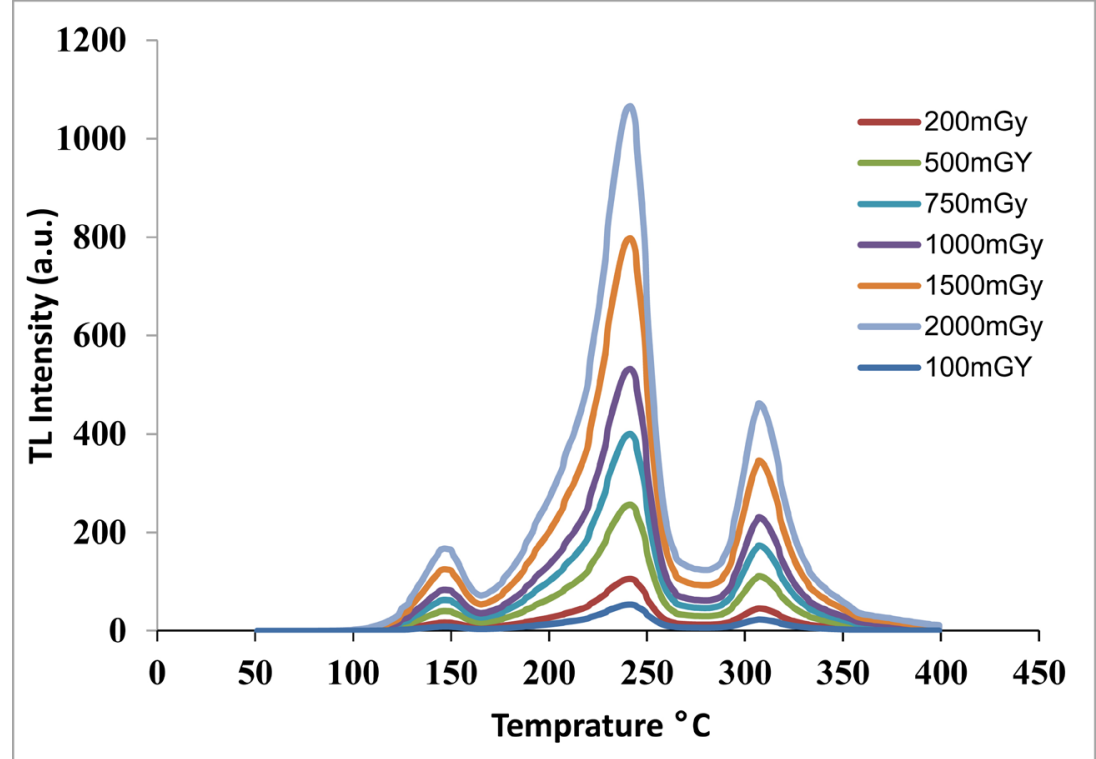

Figure 4. MTS-700 (TLD) glow curve at different doses (100, 200, 500, 750, 1000, 1500 and $2000 \mathrm{mGy}$ ) at $10^{\circ} \mathrm{C} / \mathrm{s}$ TTP.

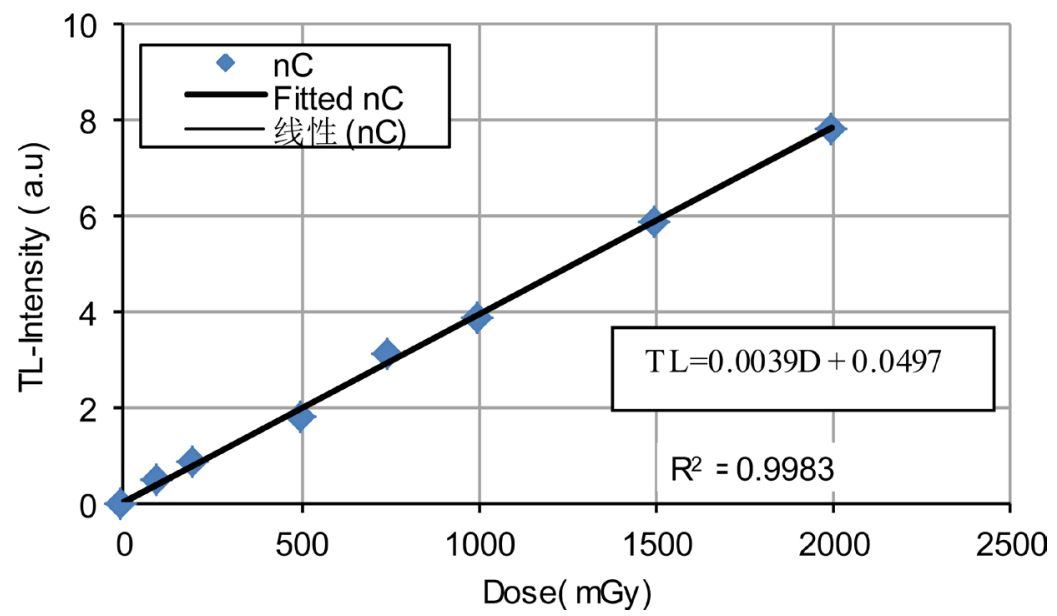

Figure 5. MTS-700 (TLD) conversion factor from (nC) to (mGy). 
units of Clinical oncology hospital, Menoufia University, Egypt shown in Table 5 and Table 6 , respectively.

The results of extremity dose measurements using MTS-700 (TLD) for nuclear medicine workers shown in Table 7 and Table 8 respectively.

\section{Discussion}

A comparison between the values of the annual ambient doses measured with surveymeter and MTS-700 (TLD) in the radiation therapy working area is shown in Table 4. The working areas are radiation therapy clinic, ${ }^{60} \mathrm{Co}$ Control room, patient waiting, long hall, worker path room, linear control, simulator control and ${ }^{60} \mathrm{Co}$ room. Values obtained by MTS-700 (TLD) are slightly higher than those obtained by surveymeter. The maximum $\%$ difference between read out of both the surveymeter and MTS-700 (TLD) did not exceed 6\%, which indicates good agreement between both of them. In addition, doses measured in the nuclear medicine unit were gathered in Table 5. Measuring sites are physician

Table 5. Comparison between radiation therapy unit annual ambient doses measured by Surveymeter and MTS-700 (TLD).

\begin{tabular}{cccccc}
\hline \multirow{2}{*}{ No. } & Site & \multicolumn{4}{c}{ Dose (mSvly) } \\
\cline { 3 - 6 } & & Survey meter & TLD (MTS-700) & Mean value \pm SD & \% Diff. \\
\hline 1 & Rad.Thera.Clinac & $1.094 \pm 0.021$ & $1.134 \pm 0.089$ & $1.114 \pm 0.014$ & 3.591 \\
2 & ${ }^{60}$ Co Control & $4.402 \pm 0.021$ & $4.494 \pm 0.175$ & $4.448 \pm 0.033$ & 2.068 \\
3 & Patient waiting & $0.601 \pm 0.021$ & $0.610 \pm 0.031$ & $0.606 \pm 0.003$ & 1.484 \\
4 & Long hall & $0.927 \pm 0.021$ & $0.995 \pm 0.096$ & $0.941 \pm 0.010$ & 2.976 \\
5 & Worker path room & $0.839 \pm 0.021$ & $0.884 \pm 0.051$ & $0.861 \pm 0.016$ & 5.223 \\
6 & Linear Control & $1.460 \pm 0.022$ & $1.053 \pm 0.131$ & $1.418 \pm 0.015$ & 2.902 \\
7 & Simulator control & $4.857 \pm 0.022$ & $5.112 \pm 0.187$ & $4.985 \pm 0 . .91$ & 5.116 \\
8 & ${ }^{60}$ Co Room & $76.536 \pm 0.114$ & 79.588 & $78.062 \pm 1.087$ & 3.910 \\
\hline
\end{tabular}

Table 6. Comparison between nuclear medicine unit Annual ambient doses measured by Surveymeter and MTS-700 (TLD).

\begin{tabular}{cccccc}
\hline \multirow{2}{*}{ No. } & Site & \multicolumn{4}{c}{ Dose (mSvly) } \\
\cline { 3 - 6 } & & Survey meter & TLD(MTS-700) & Mean value \pm SD & $\%$ Diff. \\
\hline 1 & physician station & $2.421 \pm 0.021$ & $2.584 \pm 0.102$ & $2.503 \pm 0.058$ & 6.513 \\
2 & technician station & $2.752 \pm 0.021$ & $2.866 \pm 0.002$ & $2.809 \pm 0.040$ & 4.058 \\
3 & x-ray room & $3.126 \pm 0.022$ & $3.207 \pm 0.094$ & $3.167 \pm 0.029$ & 2.558 \\
4 & injection room & $2.980 \pm 0.022$ & $3.098 \pm 0.065$ & $3.039 \pm 0.042$ & 3.883 \\
5 & preparing material & $3.718 \pm 0.022$ & $3.792 \pm 0.124$ & $3.775 \pm 0.026$ & 1.971 \\
6 & patient path room & $2.561 \pm 0.021$ & $2.754 \pm 0.112$ & $2.658 \pm 0.069$ & 7.262 \\
7 & external hall & $1.938 \pm 0.021$ & $2.076 \pm 0.035$ & $2.007 \pm 0.049$ & 6.876 \\
8 & patient waiting room & $5.503 \pm 0.023$ & 5.732 & $5.615 \pm 0.082$ & 4.077 \\
\hline
\end{tabular}


Table 7. Wrist doses (mSv) in nuclear medicine unit for preparing and injecting99m Tc persons using MTS-700 (TLD) per one week.

\begin{tabular}{ccc}
\hline \multirow{2}{*}{ TLD position } & \multicolumn{2}{c}{$\begin{array}{c}\text { Fingertip doses }(\mathrm{mSv}) \text { in nuclear medicine unit for preparing } \\
\text { and injecting }{ }^{99 \mathrm{~m}} \mathrm{Tc} \text { persons using MTS-700 (TLD) per week. }\end{array}$} \\
\cline { 2 - 3 } & Preparing & Injecting \\
\hline Right middle finger & 2.053 & 1.485 \\
Right index finger & 2.960 & 2.106 \\
Right thumb finger & 2.160 & 1.566 \\
Left middle finger & 1.885 & 1.374 \\
Left index finger & 2.847 & 2.063 \\
Left thumb finger & 2.102 & 1.524 \\
Mean value \pm SD & $2.381 \pm 0.450$ & $1.686 \pm 0.315$ \\
\hline
\end{tabular}

Table 8. Fingertip doses (mSv) in nuclear medicine unit for preparing and injecting $99 \mathrm{~m}$

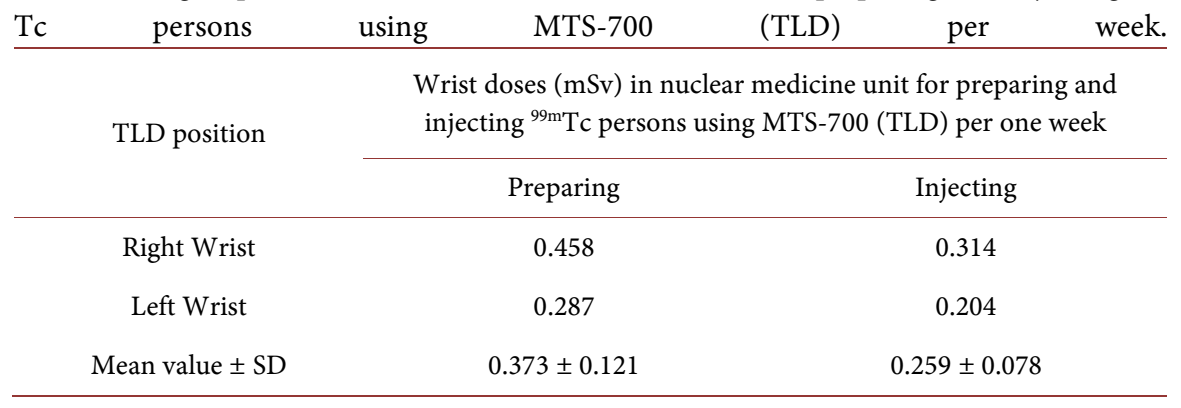

station, technician station, X-ray room, injection room, preparing material, patient path room, external hall and patient waiting room. The maximum\% difference between measurements with the above mentioned techniques not exceed $8 \%$.This difference between two different measurement methodologies may be resulted from the fact that only fifteen measurements were taken in a month with surveymeter, while MTS-700 (TLD) responded for whole time exposure. Results given in both Table 5 and Table 6 exhibit that the ambient dose values measured in this work are in compliance with the local radiation protection regulations. The design of structural shielding for radiation therapy and nuclear medicine units in the clinical oncology departmental Menoufia University, Egypt achieve the requirements and follow the guidelines of national and international commission in radiological protection.

The dose values of wrists and fingertips personal dose $\mathrm{Hp}$ (0.07) for nuclear medicine unit worker using MTS-700 (TLD) are displayed in Table 7 and Table 8.

Table 7 shows radiation doses to right and left wrists of group one (preparing ${ }^{99 \mathrm{~m}} \mathrm{Tc}$ ) and group two (injecting ${ }^{99 \mathrm{~m}} \mathrm{Tc}$ ). Radiation doses received by the wrists of radiopharmaceutical staff preparing, dispensing syringe were observed to be higher by a factor of about 1.41 than those for the administrating staff whom injected patients by ${ }^{99 \mathrm{~m}} \mathrm{Tc}$. The annual wrists doses were calculated for group 1 and 
2 by multiplying one week dose in 52.2 times (No. of weeks in one year).

Table 8 shows radiation doses to three main used fingers middle, index, and thumb of the two group workers. Radiation doses received by index fingertip of both hands were higher than radiation doses received by thumb's and middle's fingertips, these results are in agreement with the findings of Wrzesien, M. et al. [15] and indicates in-homogenies distribution of doses received by fingertips of nuclear medicine unit workers. Dose recorded by fingertips dosimeters for radiopharmaceutical staff preparing and dispensing syringe were observed to be higher by a factor of about1.44than those of the administration staff whom injected patients. The annual fingers doses for group 1 and 2 can be estimated by multiplying a factor $\mathrm{y} / \mathrm{wk} .=52.2$.

The average values of doses recorded by dosimeters placed on fingertips of middle, thumb and index fingers in one week for the two groups 1 and 2 which equal 2.381 and $1.712 \mathrm{mSv}$ respectively, the annual dose can be calculated to be 124.29 and $89.37 \mathrm{mSv}$, respectively in which the most likely equivalent dose limit $500 \mathrm{mSv}$ [20] [21] [22]. If we from the point of view consider the maximum fingertips doses which equal $2.960 \mathrm{mSv}$ and $2.106 \mathrm{mSv}$ in one week, then the annual dose will be 154.51 and $109.93 \mathrm{mSv} / \mathrm{y}$ for the two groups, respectively i.e. staff preparing will receive dose more than $3 / 10^{\text {th }}$ of the limit, which legally require routinely monitoring. With this work conditions, the equivalent dose limit will not be reached. These results are in agreement with the findings of Gauri, S. et al. [23] and Chruscielewski, W. et al. [24]. The maximum expected annual dose to the fingers appeared to be less than the annual limit ( $500 \mathrm{mSv} / \mathrm{y})$ because all of workers are on rotation and do not constantly handle radioactivity throughout the year but if the workload is increased and the protection measures stays as they are doses can reach the equivalent dose limit. So, we recommend increase the radiation protection precautions as, using the automatic injector, increasing the patient waiting area and adding a new patient bathrooms. The extent of the annual radiation exposure of the workers depends on several factors within the workplace [25] [26] [27]. These factors include, but are not limited to, the annual workload, the distribution of the workload among workers, the radiation protection practices followed by the workers, and the radiation safety facilities provided by the employers. An evaluation of how such factors affect occupational exposure will be our future study after the development of radiation therapy and nuclear medicine units of Clinical Oncology Hospital Menoufia University, Egypt.

\section{Conclusions}

MTS-700 (TLD) was subjected to several systematic investigations carried out using $\gamma$-ray doses range from 100 to $2000 \mathrm{mGy}$. These TLDs were applied in clinical radiotherapy and nuclear medicine dose measurements. Results showed that MTS-700 (TLD) has a good reproducibility, an extended range of linearity, high sensitivity, and no significant variation in response over a wide range of 
doses typically used in clinical exposures with applying the appropriate quality factor. Therefore, MTS-700 (TLD) dosimeters are capable of determining the ambient dose in radiation therapy and nuclear medicine units that received by workers.

Values of the ambient dose measured in this work are in compliance with the international radiation protection regulations. This means that design of structural shielding for radiation therapy and nuclear medicine units in these two units fulfill the requirements and follow the guidelines of national and international commission in radiological protection. Comparison between average wrists and fingertips of the thumb, index and middle finger exposure doses using MTS-700 (TLD) indicates that the fingertips can receive 6.38 and 6.51 times higher dose than those recorded by the wrist dosimeters for ${ }^{99 \mathrm{~m}} \mathrm{Tc}$ preparing and injecting radiopharmaceutical groups respectively. The radiation dose distributions on the hands of nuclear medicine unit workers are in-homogenies and complex. Measuring dose depends strongly on the position of the dosimeter, successive procedures, and an ensemble of workers. Strong variations of measuring dose are also attributed to differences in individual experiences and working habits. Herein, it can be said that this study was so important to be performed in order to provide a virtual radiation protection maps for workers, patients and visitors, in clinical oncology center at Menoufia University, Egypt this study is the first study in Egypt and the first Clinical Oncology center in Egypt has radiation protection maps for worker, patients and visitors.

\section{Acknowledgements}

The authors would like to thank Prof. Dr. A. R. Al-Sersy Head of Ionizing Radiation Dept., National Institute for Standards, Egypt for helpful discussions, Prof. Dr. N. M. Abd-Elbary Head of Clinical Oncology and Nuclear Medicine Dept., Faculty of Medicine, Menoufia University, Egypt for continuous encouragement throughout this work and all staff working in Clinical Oncology Hospital, Menoufia, Physicians, physicists, technologists and nurses.

\section{References}

[1] Federal Ministry for the Environment, Nature Conservation and Nuclear Safety (2007) Umwel-tradioaktivität und Strahlenbelastungim Jahre 2007. Annual Report of Germany.

[2] Seyedeh Sima, T.D., Mahya, A. and Ramin, M. (2017) Medical Radiation Workers' Knowledge, Attitude, and Practice to Protect Themselves against Ionizing Radiation in Tehran Province, Iran. Journal Eduction Health Promotion, 6, 58.

[3] Arslanoglu, A., Bilgin, S., Kubal, Z., Ceyhan, M.N., Ilhan, M.N. and Maral, I. (2007) Doctors' and Intern Doctors' Knowledge about Patients' Ionizing Radiation Exposure Doses during Common Radiological Examinations. Diagnostic and Interventional Radiology, 13, 53-55.

[4] European Commission (2004) European Guidelines on Radiation Protection in Dental Radiology. The Safe Use of Radiographs in Dental Practice, Issue N 136.

[5] Radiation Dosimetry (2011) From Thermoluminescence Dosimeter (TLD) to Opti- 
callystimulated Luminescence Dosimeter (OSLD) Pritsh. Journal of Radiology, 21, 107-109.

[6] McKeever, S.W., Moscovitch, M. and Townsend, P.D. (1985) Thermoluminescence Dosimetry Materials: Properties and Uses. Nuclear Technology Publishing, Ashford, Kent.

[7] Avila, O., Torres-UIIoa, C.I., Medina, L.A., TrujiIIo-Zamudio, F.E., Gamboa-deBuen L., Buenfil, A.E. and Baradan, M.E. (2011) Tl Measurement of Ambient Dose at a Nuclear Medicine Department. Radiation Measurements, 46, 1843-1846. https://doi.org/10.1016/j.radmeas.2011.06.007

[8] ICRU Report 51 (1993) Quantities and Units in Radiation Protection Dosimetry. ICRU, Bethesda.

[9] Niewiadomski, T. (1996) 25 Years of TL Dosimetry at the Institute of Nuclear Physics, Krakow. Radiation Protecton Dosimimetry, 65, 1-6. https://doi.org/10.1093/oxfordjournals.rpd.a031596

[10] Bilski, P. (2002) Lithium Fluoride: From LiF: Mg, Ti to LiF:Mg,Cu, P. Radiation Protection Dosimetry,100, 199-206.

https://doi.org/10.1093/oxfordjournals.rpd.a005847

[11] Radcard (2013) LiF:Mg,Cu, P Thermoluminescent Phosphor \& Pellets. Radcard TLD Dosemeters. http://www.radcard.pl/det/mcpn.html

[12] Obryk, B., Bilski, P., Hodyr, K. and Mika, P. (2014) High-Level TL Dosimetry for High-Temperature Environment RAD Conference. Rad 2014, Nis, Serbia, May 27-30.

[13] International Medcom, Inc. (2005) Nuclear Radiation MonitorInspector Alert Operating Manual. http://www.naturalenergyworks.info/Home.html

[14] Jankowski, J., Olszewski, J. and Kluska, K. (2003) Distribution of Equivalent Doses to Skin of the Hands of Nuclear Medicine Personnel. Radiation Protection Dosimetry, 106, 177-180. https://doi.org/10.1093/oxfordjournals.rpd.a006347

[15] Wrzesien, M., Olszewski, J. and Jankowski, J. (2008) Hand Exposure to Ionizing Radiation of Nuclear Medicine Workers. Radiation Protection Dosimetry, 130, 3325-3330. https://doi.org/10.1093/rpd/ncn057

[16] ICRU (2011) Report 85: Fundamental Quantities and Units for Ionizing Radiation. Journal of ICRU, 11, 1-31.

[17] IEC (2012) Thermoluminescence Dosimetry Systems for Personal and Environmental Monitoring of Photon and Beta Radiation. IEC 62387, Ed. 1.

[18] Chougaonkar, M.P., Kumar, M. and Bhatt, B.C. (2012) Testing of Phosphors for Their Use in Radiation Dosimetry: Detailed Procedure and Protocol. International Journal of Luminescence and Applications, 2, 194-222.

[19] McKinlay, A.F. (1981) Thermoluminescence Dosimetry-Medical Physics Handbooks 5. Adam Hilger Ltd., Bristol.

[20] ICRP (2007) Publication No. 103: The 2007 Recommendations of the International Commission on Radiological Protection. Annals of the ICRP, 37, 2-4.

[21] Mattsson, S. and Hoeschen, C. (2013) Radiation Protection in Nuclear Medicine. Springer-Verlag, Berlin Heidelberg.

[22] Shaw, P.V., Croual, P. and Coeck, R.M. (2015) Education and Training in Radiation Protection: Improving ALARA Culture. Journal of Radiological Protection, 35, 223-227. https://doi.org/10.1088/0952-4746/35/1/223

[23] Pant, G.S., Sanjay Sharma, K. and Gaura Rath, K. (2006) Finger Doses for Staff Handling Radiopharmaceuticals in Nuclear Medicine. Journal of Nuclear Medicine 
Technology, 34, 3.

[24] Chruscielewski, W., Olszewski, J., Jankowski, J. and Cygan, M. (2002) Hand Exposure in Nuclear Medicine Workers. Radiation Protection Dosimetry, 101, 229-232. https://doi.org/10.1093/oxfordjournals.rpd.a005973

[25] Covens, P., Berus, D., de Mey, J., et al. (2012) Mapping Very Low Level Occupational Exposure in Medical Imaging: A Useful Tool in Risk Communication and Decision Making. European Journal of Radiology, 81, 962-966. https://doi.org/10.1016/j.ejrad.2012.06.014

[26] Linet, M.S., Kim, K.P. and Miller, D.L. (2010) Historical Review of Occupational Exposures and Cancer Risks in Medical Radiation Workers. Radiation Research, 174, 793-808. https://doi.org/10.1667/RR2014.1

[27] Al Haj, A.N. and Lagarde, C.S. (2002) Statistical Analysis of Historical Occupational Dose Records a Large Medical Centre. Health Physics, 83, 854-860.

https://doi.org/10.1097/00004032-200212000-00013 